\title{
Christophe Cave et Simon Davies (dir.), Les Vies de Voltaire: discours et représentations biographiques, $\mathrm{XVIII}{ }^{\mathrm{e}}-\mathrm{XXI} \mathrm{e}^{\mathrm{e}}$ siècles
}

\section{Valentina Ponzetto}

\section{(2) OpenEdition}

\section{Edizione digitale}

URL: http://journals.openedition.org/studifrancesi/7577

DOI: 10.4000/studifrancesi. 7577

ISSN: 2421-5856

\section{Editore}

Rosenberg \& Sellier

\section{Edizione cartacea}

Data di pubblicazione: 1 décembre 2009

Paginazione: 626-627

ISSN: 0039-2944

\section{Notizia bibliografica digitale}

Valentina Ponzetto, «Christophe Cave et Simon Davies (dir.), Les Vies de Voltaire: discours et

représentations biographiques, xVIII -xxle siècles», Studi Francesi [Online], 159 (LIII | III) | 2009, online dal 30 novembre 2015, consultato il 09 janvier 2021. URL: http://journals.openedition.org/studifrancesi/7577 ; DOI: https://doi.org/10.4000/studifrancesi.7577

Questo documento è stato generato automaticamente il 9 janvier 2021.

\section{(c) (i) $\odot$}

Studi Francesi è distribuita con Licenza Creative Commons Attribuzione - Non commerciale - Non opere derivate 4.0 Internazionale. 


\title{
Christophe Cave et Simon Davies (dir.), Les Vies de Voltaire: discours et représentations biographiques, $\mathrm{XVIII}^{\mathrm{e}}$ - XXI $I^{\mathrm{e}}$ siècles
}

\author{
Valentina Ponzetto
}

\section{NOTIZIA}

CHRISTOPHE CAVE et SIMON DAVIES (dir.), Les Vies de Voltaire: discours et représentations biographiques, XVIII ${ }^{\mathrm{e}}$ XXI ${ }^{\mathrm{e}}$ siècles, Oxford, Voltaire Foundation, 2008 (SVEC 2008:04), pp. 478.

1 Come affrontare, oggi, la biografia di un autore assurto al rango di vera e propria icona qual è Voltaire? Come rapportarsi alle multiformi e contraddittorie immagini che ne sono state fornite nel corso di più di due secoli? E soprattutto «à quelle société et à quelles valeurs peut-elle encore nous renvoyer»? A questi interrogativi offrono una variegata gamma di risposte gli interventi dei 28 studiosi riuniti nel presente volume, risultato dei lavori dell'omonimo convegno internazionale tenutosi a Grenoble nel settembre 2004.

2 L'introduzione di Christophe CAVE (pp. 1-25) e la prima sezione, «Problématiques», mettono in evidenza le problematiche legate alla definizione, alla natura e alla metodologia d'approccio del genere biografico che, come mostra Jean SGARD (Poétiques des vies particulières, pp. 29-41), nasce nella sua concezione moderna proprio nel XVIII secolo, distaccandosi dal modello agiografico e pedagogico per accostarsi alla Storia e soprattutto al romanzo grazie alla sua ricerca del «détail signifiant». In una prospettiva letteraria la biografia, o «vie de» secondo la denominazione più corrente all'epoca, si situa dunque alla frontiera fra verità e finzione, ma anche, come mostrano molti degli interventi del volume, fra ricerca di un'obbiettività scientifica e intenti polemici, fra 
tentazione dell'aneddoto e ricerca di un percorso significativo in un determinato contesto storico e sociale. Come mostra lo storico Daniel Roche attraverso la sua analisi delle peregrinazioni di Voltaire in Europa, una biografia «doit à la fois faire la part du hasard d'une vie et référer le parcours du sujet à des déterminations sociales» (Voltaire, $d u$ voyage à la philosophie, pp. 43-60). Altri nodi problematici messi in evidenza sono l'affidabilità delle testimonianze tratte dalla corrispondenza, il rischio della falsificazione dei documenti e il valore da dare agli stereotipi forgiatisi nel corso del tempo, di cui è necessario decrittare il sottotesto strategico o ideologico.

3 In questa prospettiva l'intera seconda sezione del libro è dedicata, riprendendo la definizione di Roland Barthes, ai «Biographèmes», ovvero a quei dettagli di una vita, spesso eredi dell'antica tradizione dell'aneddoto, che hanno subito un processo di cristallizzazione simbolica migrando, per così dire, da una biografia all'altra, in un susseguirsi di stratificazioni discorsive interessanti da analizzare. I biografemi presi in considerazione sono, nell'ordine: l'infanzia di Voltaire e i suoi rapporti con i genitori e l'educazione (Anne-Marie MERCIER-FAIVRE, Récits d'enfance: le petit Arouet dans l'ombre du grand Voltaire, pp. 63-80), l'episodio della bastonata ordinata dal cavaliere di Rohan (Henri DURANTON, Voltaire bâtonné: ni Arouet ni Figaro, les avatars d'une ténébreuse affaire, pp. 81-105), le immagini di un Voltaire bugiardo, avaro o coprofago tratte dalla corrispondenza e deformate a fini polemici (Christophe CAVE, Lettre et biographie: Voltaire "peint par lui-même", pp. 107-123), il tentativo di fissare un'iconografia dello scrittore attraverso statue e dipinti (Muriel CATTOOR, Virevoltant Voltaire: images et statues de Voltaire, pp. 125-133). E si può ancora aggiungere all'elenco l'articolo di Christiane MERVAUD sull'«avanie de Francfort», ovvero l'arresto di Voltaire e di sua nipote per ordine di Federico II (Des "Mémoires pour servir à la vie de M. de Voltaire" aux "Vies" de Voltaire: l'avanie de Francfort, pp. 155-169) e quello di Nicholas CRONK sul soggiorno a Cirey in compagnia di Mme du Châtelet come appare nei Mémoires di Longchamps ((Ré)écrire les années de Cirey, ou du bon usage des anec-dotes de Longchamp, pp. 171-187), episodi privilegiati e dotati di una fortuna a sé stante.

Questi ultimi due saggi, a dire il vero, sono inseriti nella terza sezione, "Autobiographies», che copre uno spettro più ampio di quanto il titolo non sembri indicare, poiché comprende anche le testimonianze su Voltaire redatte non già da lui stesso ma dai suoi segretari Longchamps, Collini e Wagnière (Nicholas CRONK, cit.; Dinah RIBARD, Secrétaire, témoin, auteur: les "vies" de Voltaire par ses secrétaires, pp. 189-199) o, all'inverso, un intervento su Voltaire biografo delle vite altrui. In Réflexions sur l' "Histoire de Charles XII": biographie et autobiographie (pp. 137-143), Simon DAVIES mostra come biografia e autobiografia siano strettamente legate, poiché riflettendo sul destino dei sovrani Voltaire comincia a meditare sulla propria esistenza e sulle strategie narrative adatte a raccontare una vita. La particolarità della scrittura più propriamente autobiografica di Voltaire è messa in luce da Jean GoldzinK che ne sottolinea l'inedito carattere comico, rilevando altresì come questa caratteristica fa dei Mémoires pour servir à la vie de M. de Voltaire «le dispositif le plus radicalement anti-rousseauiste jamais imaginé» (La comédie des Mémoires: du comique en autobiographie, pp. 145-153).

5 La quarta e più ampia sezione del volume, «Biographies», affronta il quadro delle numerose opere a carattere biografico dedicate a Voltaire apparse dalla metà del Settecento ad oggi, sottolineando alcuni momenti forti e delle linee guida che permettono di individuare in maniera criticamente fondata diverse fasce cronologiche successive. 
6 Finché Voltaire stesso era ancora in vita, ovviamente, ogni discorso biografico non poteva essere che frammentario e di parte. Gli articoli di Graham Gargett, Olivier Ferret e Anne-Sophie Barrovecchio ne offrono tre ottime testimonianze, trattando rispettivamente dei Mémoires de M. de Voltaire di Goldsmith, un ammiratore entusiasta dello scrittore (Graham GARGETT, Olivier Goldsmith et ses "Mémoires de M. de Voltaire", pp. 203-222), della Vie polémique di Sabatier de Castres, opera al contrario quanto mai denigratoria (Olivier FERRET, Ecrire une "vie polémique» de Voltaire: les biographies de Sabatier de Castres, pp. 223-238), e del Testament politique de M. de $V^{* * *}$ di Marchand, che opera per pastiche e imitazione cercando nella figura e nell'opera di Voltaire spunto per riflessioni morali (Anne-Sophie BARROVECCHIO, Tester ou témoigner? Le discours biographique de l'avocat Marchand sur Voltaire: deux destins pour une seule vie, p. 239-252).

7 La morte di Voltaire segna l'inizio di un nuovo periodo che dura all'incirca fino alla Rivoluzione: quello degli elogi e delle «fictions valorisantes», opera di philosophes militanti che tendono a ridurre lo scrittore ad uno stereotipo simbolico, come ad esempio quello di «apôtre de la tolérance» analizzato da Otto H. SELLES (Voltaire, «apôtre de la tolérance»: les "Eloges" de Palissot et La Harpe (1778-1780), pp. 255-267), o addirittura a farne un comodo schermo su cui proiettare polemiche e prese di posizione politiche di scottante attualità, come mostrato da Huguette KRIEF (Triomphe et apothéose de Voltaire: les enjeux d'une critique biographique pendant la Révolution, pp. 283-298). Anche il teatro rivoluzionario non perde l'occasione di mettere in scena l'icona semplificata e militante di un Voltaire triomphant come mostra l'omonimo dramma antireligioso di Cloots analizzato da Yves CITTON (La propagande du dernier souffle: la mort de l'athée dans "Voltaire triomphant"d'Anacharsis Cloots, pp. 299-319). E' intanto iniziata l'epoca delle prime grandi biografie dotate di rigore scientifico: quella di Duvernet (1786), la monumentale Histoire littéraire de M. de Voltaire di Luchet (1780), erede del genere dell'examen des cuvvres più che della vera e propria biografia e qui presentata da Didier MASSEAU ( $L$ ' «Histoire littéraire de M. de Voltaire» du marquis de Luchet: mise en scène et enjeu d'un discours biographique et critique, pp. 323-335), e la Vie de Voltaire di Condorcet (1787), un «manifesto filosofico» che però rifugge dall'agiografia, come sottolinea Charles COUTEL (La "Vie de Voltaire" de Condorcet, pp. 337-343).

8 Le posizioni cambiano radicalmente a partire dalla Restaurazione: ferma restando la convinzione del ruolo storico fondamentale rappresentato da Voltaire il giudizio su tale ruolo si fa pesantemente negativo: Lepan e Mazure discreditano sistematicamente l'uomo e l'opera, in un processo polemico di demonizzazione che forgia l'immagine del "Voltaire anticristo" dominante per tutto il secolo (Raymond TROUSSON, Edouard Marie Lepan: un biographe de Voltaire en 1817, pp. 345-359; Jean-Noël PASCAL, Deux portraits de Voltaire en antéchrist au temps de la Restauration: note sur Mazure (1821) et Lepan (1823), pp. 361-373). Persino La Harpe, in gioventù amico e grande ammiratore di Voltaire, rivede nettamente le sue posizioni alla luce di un ritrovato cattolicesimo all'inizio dell'Ottocento (Rémy LANDY, La Harpe à propos de Voltaire: un biographe intermittent, pp. 269-281). All'altro capo del secolo i virulenti pamphlets di Eugène de Mirecourt distillano in maniera particolarmente rivelatrice l'essenza del discorso di condanna tenuto dalla destra cattolica del tempo (Valérie ANDRÉ, Eugène de Mirecourt: "biographe" de Voltaire, pp. 375-384).

Più accademico ma solo apparentemente meno parziale, il xx secolo rovescia ancora una volta l'immagine di Voltaire, facendone in sostanza il padre della tolleranza e della 
laicità francese e il modello dello scrittore engagé. E' quanto mostrato da Béatrice BOMELRAINELLI, che analizza l'evoluzione degli elementi salienti della biografia voltairiana in 40 manuali scolastici apparsi fra il 1841 e il 2003 (Le rire du roi Voltaire: 160 ans de biographies scolaires, pp. 407-422).

10 Miti, immagini simboliche e proiezioni intellettuali si cristallizzano intorno alla figura di Voltaire anche in nuove opere di finzione di diverso genere analizzate in chiusura del volume: romanzi (Haydn MASON, Nancy Mitford, "Voltaire in love": une vraie biographie?, pp. 387-395), dialogues des morts (J. Patrick LEE, Voltaire dans les dialogues des morts et conversations imaginaires en langue anglaise au vingtième siècle, pp. 397-406), film (Denis REYNAUD, Voltaire au cinéma, pp. 423-431) e opere teatrali (Hervé LoICHEMOL, Porter la vie (de Voltaire) au théâtre, pp. 433-436). Completando le biografie tradizionali, tali realizzazioni artistiche ci rammentano che, come sottolinea Christophe Cave, «une vie n'a de valeur pour nous qu'en tant qu'elle donne à penser, agir, rêver le présent». 